\title{
Cold pressor test in spinal cord injury-revisited
}

\author{
Michèle Hubli ${ }^{1,2} \cdot$ Doris Bolt $^{3} \cdot$ Andrei V. Krassioukov ${ }^{1,4,5}$
}

Received: 2 June 2017 / Revised: 8 November 2017 / Accepted: 11 November 2017 / Published online: 20 December 2017

(c) International Spinal Cord Society 2018

\begin{abstract}
Study design Systematic review.

Objectives A spinal cord injury (SCI) commonly results in alterations of cardiovascular physiology. In order to investigate such alterations, the cold pressor test (CPT) has been used as an established challenge test. This review summarizes the basic physiology underlying a CPT, discusses potential mechanisms responsible for abnormal pressor responses following SCI, and highlights the utility of CPT in the SCI population.

Setting Canada and Switzerland.

Methods We have completed a comprehensive review of studies that have investigated the effect of foot or hand CPT on hemodynamic indices in individuals with SCI.

Results Depending on the level of spinal cord lesion and the location of cold application, i.e., above or below the lesion, mean arterial pressure typically increases (ranging between 4 and $23 \mathrm{mmHg}$ ), while heart rate responses demonstrated either a decrease or an increase (ranging between -4 and $24 \mathrm{bpm}$ ) during CPT. The increase in blood pressure during foot CPT in high-level lesions might not necessarily be attributed to a physiological CPT response as seen in able-bodied individuals, but rather due to a reflexic sympathetic discharge below the level of lesion, known as autonomic dysreflexia.

Conclusions Further investigations in a wider range of individuals with SCI including incomplete injuries might be helpful to examine the ability of CPT assessing the integrity of the autonomic nervous system following SCI. Furthermore, additional autonomic tests are needed to emphasize the integrity of autonomic pathways and to account for the complexity of the autonomic nervous system.
\end{abstract}

\section{Introduction}

The autonomic nervous system controls cardiovascular function, including peripheral vasomotor tone, blood pressure (BP), cerebral and coronary blood flow, heart contractility, and heart rate (HR). A spinal cord injury (SCI) can

$\triangle$ Andrei V. Krassioukov

krassioukov@icord.org

1 International Collaboration on Repair Discoveries (ICORD), Blusson Spinal Cord Centre (BSCC), University of British Columbia, Vancouver, BC, Canada

2 Spinal Cord Injury Center, Balgrist University Hospital, University of Zurich, Zurich, Switzerland

3 Institute of Physiotherapy, Zurich University of Applied Science, Winterthur, Switzerland

4 Department of Medicine, Division of Physical Medicine and Rehabilitation, University of British Columbia, Vancouver, BC, Canada

5 GF Strong Rehabilitation Centre, Vancouver, BC, Canada alter cardiovascular function and various tests have been used to assess vasomotor tone and BP regulation in this patient population [1-3]. Most of these clinical tests measure the end-organ response to different physiological perturbations because the anatomic location of the cardiovascular autonomic nervous system renders it rather inaccessible to simple direct physiological testing [4]. One of these routinely used tests is the cold pressor test (CPT) reflecting a provocative measure of autonomic vascular regulation eliciting an autonomic hemodynamic phenomena, such as BP and HR increase [5]. During a CPT, subjects are instructed to immerse the hand or foot in a container of ice water for $1-3 \mathrm{~min}$, while their BP and HR are monitored. The application of cold first activates afferent sensory pathways and next triggers sympathetic nervous system responses, resulting in increase of BP and HR in healthy individuals [4]. In disease conditions where afferent and/or efferent neuronal circuits and pathways are damaged, the sympathetic vasomotor response is abnormal and, therefore, BP and HR responses to CPT can vary significantly. As an SCI can result in a disruption of neuronal 
Table 1 Subject characteristics and hemodynamic CPT outcomes

\begin{tabular}{|c|c|c|c|c|c|c|c|}
\hline Author & Subjects & Level of lesion & AIS & Duration of lesion & CPT & $\Delta \mathrm{BP}(\mathrm{MAP})$ & $\Delta \mathrm{HR}(\mathrm{bpm})$ \\
\hline \multirow[t]{2}{*}{ Ogata et al. (2012) } & 7 tetra & C6 & A & $>38$ months & Foot & Tetra $14 \pm 9 *$ & Tetra $-4 \pm 4^{*}$ \\
\hline & 5 para & T5/T6 & & & & Para $6 \pm 6$ & Para $-8 \pm 2 *$ \\
\hline \multirow[t]{4}{*}{ Previnaire et al. (2012) } & 14 tetra & Above T6 & A & $>4$ months & Hand & Tetra 5 (SBP) & N/A \\
\hline & 12 para & & & & & Para 7* (SBP) & \\
\hline & & & & & Foot & Tetra $7 *(\mathrm{SBP})$ & N/A \\
\hline & & & & & & Para 4* (SBP) & \\
\hline \multirow[t]{2}{*}{ Catz et al. (2008) } & 11 tetra & $\mathrm{C} 4-\mathrm{C} 7$ & Tetra: A/B & $>3$ months & Hand & Tetra $9 *$ & Tetra $4 *$ \\
\hline & 10 para & $\mathrm{T} 4-\mathrm{T} 6$ & para: A & & & Para 7 & Para $10 *$ \\
\hline \multirow[t]{2}{*}{ Catz et al. (2007) } & 11 tetra & $\mathrm{C} 4-\mathrm{C} 7$ & Tetra: A/B & $>3$ months & Foot & Tetra $16^{*}$ & Tetra $-8 *$ \\
\hline & 10 para & T4-T6 & Para: A & & & Para 4 & Para $-7 *$ \\
\hline Mizushima et al. (2003) & 8 tetra & $\mathrm{C} 6-\mathrm{C} 8$ & A & $>2$ years & Foot & $20 *$ & -2 \\
\hline \multirow[t]{2}{*}{ Winchester et al. (2000) } & $4 \mathrm{BSS}$ & $\mathrm{C} 5, \mathrm{C} 5, \mathrm{~T} 4, \mathrm{~T} 8$ & N/A & $>6$ months & Foot & Intact side $23^{*}$ & Sens intact $24 *$ \\
\hline & & & & & & Not intact side $-{ }^{1}$ & Sens not intact $-^{1}$ \\
\hline \multirow[t]{2}{*}{ Lehmann et al. (1989) } & 20 tetra & $\mathrm{C} 5-\mathrm{C} 8$ & $\mathrm{~A} / \mathrm{B}$ & $>6$ months & Hand or forehead & Tetra $\downarrow 1$ & Tetra $\uparrow^{1}$ \\
\hline & 20 para & T10-L1 & & & & Para $\uparrow * 1$ & $\operatorname{Para} \uparrow *^{1}$ \\
\hline \multirow[t]{2}{*}{ Pollock et al. (1950) } & 11 tetra & N/A & $\mathrm{A} / \mathrm{B}$ & N/A & Hand & 2 (SBP) & N/A \\
\hline & & & & & Foot & $18 *(\mathrm{SBP})$ & N/A \\
\hline
\end{tabular}

AIS American spinal injury association Impairment Scale, BSS Brown-Séquard syndrome, $C$ cervical, $C P T$ cold pressor test, $N / A$ not available, $T$ thoracic, $\uparrow$ increase, $\uparrow$ decrease, - unchanged

*Significant ${ }^{1}$ Exact BP/HR value not available

pathways involved in the control of spinal sympathetic circuits, the outcome of a CPT in individuals with SCI might be altered. Several publications have investigated CPT responses in individuals with SCI [6-13]. Depending on the lesion level of an SCI and the location of cold application, the CPT responses can vary enormously. Besides, the CPT might be a useful tool to investigate the remaining level of autonomic control after a lesion to the spinal cord.

The aim of this study was to therefore conduct a critical literature review of the autonomic cardiovascular responses to CPT in individuals with SCI with particular analysis of the effect of lesion level and CPT location.

Furthermore, we will discuss methodological considerations for the potential use of the CPT as a useful test in assessing completeness of injury to spinal autonomic pathways following SCI.

\section{Methods}

This literature review is based on a literature search performed using Pubmed and Medline search engines. Only peer-reviewed articles published until December 2016 were included. Inclusion criteria were human studies including SCI in English language. The literature search was performed using the keywords outlined as follows: "cold pressor test" AND ("SCI" OR "paraplegia" OR "tetraplegia").

\section{Results}

Eight studies have investigated the effect of hand CPT [8, $10,12,13]$ and foot CPT $[6,7,9-11,13]$ on cardiovascular outcomes, such as BP and HR, in individuals with SCI. Furthermore, several studies also examined responses in cerebral blood flow velocity and cerebrovascular resistance [8, 9], endocrine responses [7], calf vascular resistance [14], skin temperature [7], and tissue oxygenation [6] during CPT. For the purpose of this review we are, however, focusing only on BP and HR as they were the most common CPT outcomes across all CPT studies in individuals with SCI.

Subjects characteristics and hemodynamic outcomes of all CPT studies are shown in Table 1. Studies did investigate tetraplegic (C4-C8) and/or high-level paraplegic (T1-T6) individuals. Only one study included individuals with Brown-Séquard syndrome with level of lesion from C5 to T8 [11] and another included only individuals with lowlevel paraplegia (T10-L2). The minimum duration of lesion was 3 months. All studies were done in subjects suffering a sensorimotor complete lesions (American Spinal Injury Association (ASIA) Impairment Scale (AIS) A), except of three studies where some individuals with sensory incomplete tetraplegia (AIS B) were investigated [8, 9, 13]. The studies did investigate either foot or hand CPT or both. In the following parts, first the physiological basis of a CPT response in healthy controls will be explained in order to 
Fig. 1 Schematic drawing of anatomic pathways involved in a hand CPT response. Stimulation of afferent nerves will transmit via dorsal root ganglion into the spinal cord and synapse with spinal and supraspinal structures, such as the thalamus and subsequently the vasomotor brainstem regions. This results in activation of the efferent pathway through sympathetic preganglionic neurons (SPNs). The SPNs synapse on the sympathetic postganglionic neuron and relay impulses to the effector organs: blood vessels and heart (adapted with permission from Berger et al. [57]) (Color figure online)

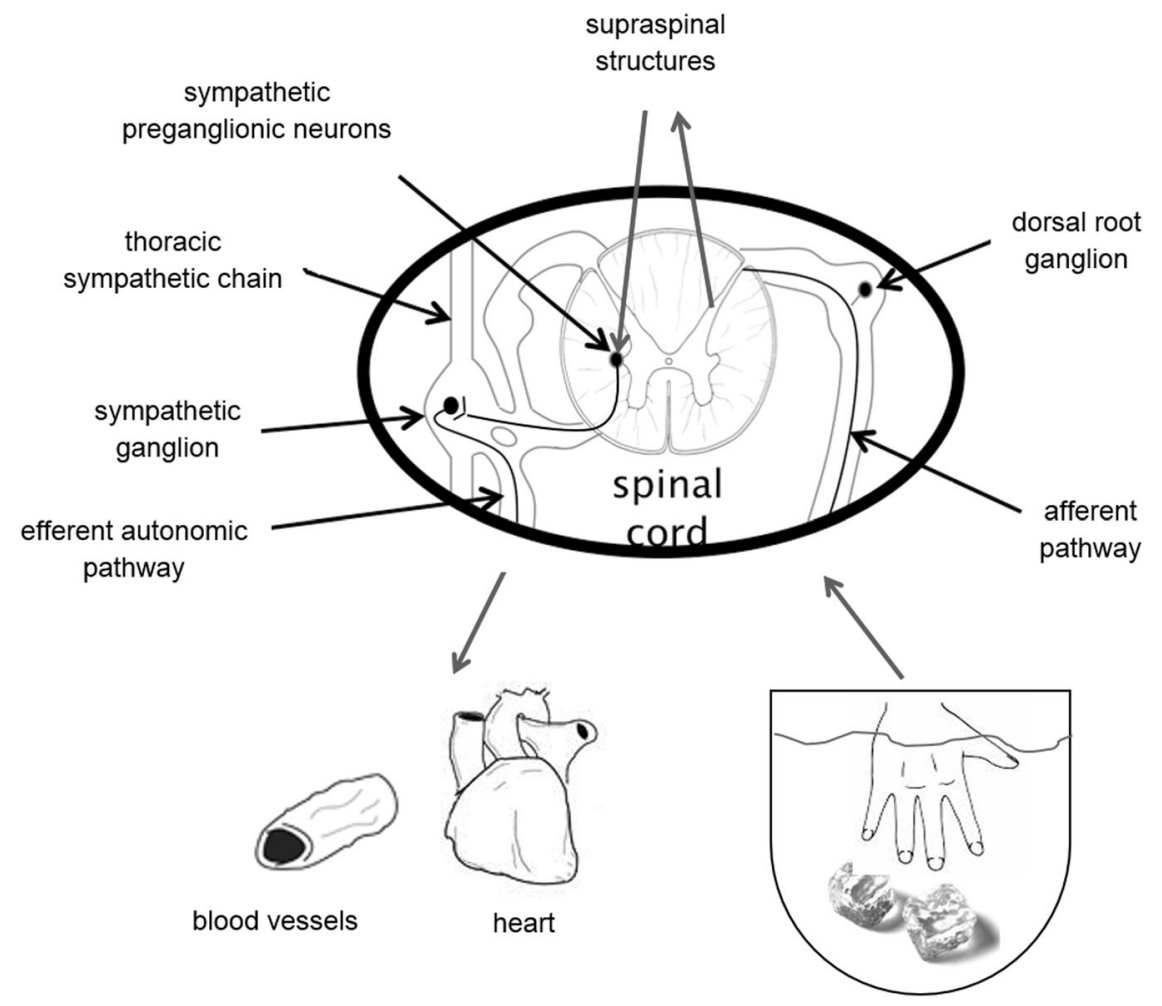

supraspinal

sympathetic

orsal root ganglion

athway

then better discuss the results of foot CPT and hand CPT. As the level of lesion plays a major role for the CPT outcome, both hand and foot CPT sections address tetra- and paraplegia consecutively.

\section{Discussion}

In general, the cold stimulus applied during a CPT induces an alteration of vascular tone, i.e., vasoconstriction, caused by two different mechanisms: (i) a local myogenic response to the cold in the arteries and arterioles of the immersed limb and (ii) a generalized vascular response due to activation of the sympathetic nervous system as described below. The myogenic response is an intrinsic mechanism inherent to the vasculature itself and leads to smooth muscle contraction [15]. This significantly reduces the volume of blood able to pass through the lumen which reduces blood flow through the blood vessel and increases BP.

The generalized vascular response is a result of the activation of nociceptors exposed to the cold and sensory afferent nerve fibers which bring information to the spinal cord and ultimately trigger activation of the spinal sympathetic preganglionic neurons (SPNs) resulting in increase of peripheral sympathetic activity. The increase in sympathetic activity leads to increased peripheral resistance through vasoconstriction of peripheral blood vessels, as well as increased HR and cardiac contractility (see Fig. 1). Consequently, BP increases, as it is the result of cardiac output
(HR and cardiac contractility) and total peripheral resistance. In most cases the cold stimulus is applied to either the hand or/and the foot which activates sensory nerve fibers at C6-T1 and/or L4-S1 spinal level, respectively. The afferent nerve fibers enter the spinal cord via the dorsal root and run within the spinothalamic tract to supraspinal structures, such as the thalamus, and subsequently activate the medullary vasomotor center, i.e., the $\mathrm{C} 1$ cells in the rostroventrolateral medulla (RVLM) [16]. Findings from an experimental study in rats confirm the important contribution of glutamatergic receptors located in the RVLM and the spinal cord, as well as $\gamma$-aminobutyric acid receptors in the nucleus ambiguus to the pressor response [17]. The RVLM is the primary vasomotor center in the brainstem and controls cardiovascular function [18]. The efferent sympathetic pathway leaving from the RVLM has two neurons interposed between the central nervous system and the innervated target organ. The first neuron is the SPN, its axon passes to the periphery within the ventral root of the spinal cord (T1-L2). The SPNs synapse in sympathetic ganglions located in the sympathetic vertebral chain with second neurons, called postganglionic neurons. The postganglionic neurons, as the efferent autonomic pathways, lie entirely outside the central nervous system and relay impulses to the effector organ, which in the case of a CPT are the heart and blood vessels [19]. The sympathetic innervation of the heart and upper limb blood vessels origins from $\mathrm{T} 1$ to $\mathrm{T} 5$ spinal levels, while the ones for blood vessels of the splanchnic bed and lower limbs stems from T6 to L2 (see Fig. 3) [19]. 


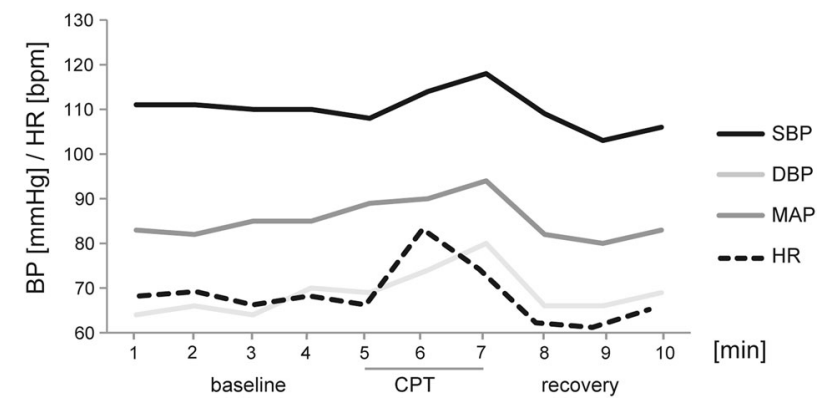

Fig. $2 \mathrm{BP}$ and HR changes during hand $\mathrm{CPT}$ in an able-bodied individual (female, 27 years). BP and HR have been discretely monitored every minute. BP increases during hand CPT with peak in the second minute of hand CPT $(\Delta \mathrm{SBP} 10 \mathrm{mmHg} ; \Delta \mathrm{DBP} 16 \mathrm{mmHg} ; \Delta \mathrm{MAP} 11$ $\mathrm{mmHg}$ ). HR increases during hand CPT with peak in the first minute of CPT ( $\triangle \mathrm{HR} 17 \mathrm{bpm}$ ) (unpublished data from our laboratory)

In the sympathetic nervous system, the heart and vasculature receive adrenergic postganglionic innervation through the neurotransmitter norepinephrine. The effects of norepinephrine are determined by two types of postsynaptic receptors, known as alpha and beta receptors. The beta receptors are located in the heart and their activation elicits an increase in HR and force of contraction. In the vasculature, activation of alpha receptors elicits vasoconstriction of peripheral blood vessels which increases peripheral resistance and ultimately increases BP [19].

The baroreflex is a complex negative feedback system and plays a major role in CPT responses. Arterial stretch receptors in the carotid and aortic arch provide surrogate information on current BP to the nucleus tractus solitarius, which then modulates both the sympathetic and parasympathetic autonomic divisions. Two systems work together as one reflex system to provide short-term regulation of BP and HR. The first, a low-pressure system, enhances sympathetic nervous system activity to increase BP and HR, while the second, a high-pressure system, enhances parasympathetic nervous system activity to decrease BP and HR [20]. During a CPT, BP increases and therefore the highpressure system of the baroreflex enhances parasympathetic nervous system activity resulting in subsequent downregulation of $\mathrm{BP}$ and HR [21]. In more detail, during high $\mathrm{BP}$ phases the stretch receptors (arterial baroreceptors) of the distended carotid and aortic arterial walls generate action potentials traveling as neuronal signals from the carotid bulb via the glossopharyngeal nerve (cranial nerve IX), and the aortic arch via the vagal nerve (cranial nerve $\mathrm{X}$ ), to the nucleus of the solitary tract in the medulla oblongata [19]. This transmission of information on systemic BP leads to an adequate adaptation of BP maintenance through an increased vagal tone and a sympathetic inhibition. Consequently this results in decreased vascular tone, venous return, cardiac contractility, and HR [22].

\section{CPT in able-bodied individuals}

The physiological CPT response in able-bodied individuals is an increase in systolic and diastolic BP of about 10-20 mmHg compared to baseline as well as an increase in HR (see Fig. 2) [16]. The maximum BP is normally reached within 1-2 min of cold application [23] and it returns to baseline within 2 min after removal of the cold stimulus [7]. There are no significant differences between the foot and hand CPT in able-bodied individuals [24, 25]. In addition, CPT reliability analyses indicate that the hand and foot responses are reliable over a 2 -week test-retest interval [25].

\section{CPT in neurological disease conditions}

CPTs have been used in a variety of disease conditions affecting the peripheral or central nervous system. A physiological CPT response is relying on fully intact sensory (afferent) and autonomic (efferent) nervous systems with functional central and peripheral, neuronal circuits. Hence, altered CPT responses are expected in neurological disease conditions with impaired central and/or peripheral neuronal circuits. Attenuated CTP responses have been found in various neurological disorders or conditions affecting various parts of nervous system including Parkinson's diseases (PD), multiple system atrophy, multiple sclerosis (MS), diabetic neuropathy, and other conditions. However, it has to be mentioned that the mechanisms underlying the abnormal CPT responses in these neurological conditions can vary significantly.

In individuals with $\mathrm{PD}$, an attenuated increase of mean arterial pressure (MAP) and cerebral blood flow velocity in response to CPT has been observed and attributed to central and peripheral impairments in the sympathetic tone driving the MAP [26]. The almost ubiquitous neuronal loss and presence of Lewy bodies (a marker for neuronal degeneration in PD patients) can be found within various parts of the nervous system, including various components of the autonomic nervous system. Lewy bodies can commonly be found within the hypothalamus, sympathetic system (intermediolateral nucleus of the thoracic cord and sympathetic ganglia), and parasympathetic system (dorsal vagal and sacral parasympathetic nuclei) [27]. In addition, there is evidence that drug therapy can contribute to secondary dysautonomia in PD, as for instances medication with dopamine agonists can exacerbate orthostatic hypotension [28].

MS is a chronic inflammatory demyelinating disease affecting the central nervous system and it is manifested by inflammation, demyelination, axonal loss, and gliosis in the brain and the spinal cord. This condition affects white 
matter tracts within various regions of the central nervous system (the spinal cord, brainstem, hypothalamus, and the cerebral cortex) which are critical for various functions including autonomic function [29]. It has been suggested that demyelination and axonal loss following a relapse may disrupt supraspinal autonomic circuits or interfere with spinal autonomic pathways [30]. In addition, diminished integrity of sensory pathways affects the proper processing of sensory information in patients with MS. Therefore, individuals with MS have demonstrated attenuated BP and HR responses to CPT [31].

In individuals with long standing diabetes mellitus, numerous investigators also demonstrated significant autonomic dysfunctions with diminished BP responses to CPT [32, 33]. However, the underlying pathophysiology of attenuated CPT responses in this population is mainly due to an impaired peripheral nervous system as a result of development of diabetic peripheral neuropathies [34]. Diabetes is considered one of the most common causes of small fiber neuropathy in which sensory symptoms, including pain and autonomic dysfunctions, frequently dominate the clinical picture.

\section{CPT in individuals with SCI}

\section{Changes in cardiovascular control following SCI}

Damage to the spinal cord can lead to disruption of supraspinal control over spinal sympathetic circuitries, such as SPNs. Depending on the level and severity of the spinal lesion, central sympathetic tone is lost or partially decreased, which leads to various severity of cardiovascular dysfunctions [35]. Although peripheral myogenic tone is preserved, it is inadequate at maintaining arterial $\mathrm{BP}$ following high thoracic or cervical SCI [36]. The segmental sympathetic innervations are particularly important for the understanding of cardiovascular control in individuals with SCI (see Fig. 3). The loss of sympathetic tonic activity to a significant portion of peripheral blood vessels plays a major role in the development of low resting arterial BP and orthostatic hypotension; hence, those individuals with the highest (cervical) injuries exhibit the most severe orthostatic hypotension and the lowest resting arterial BP [37].

Next to persistent hypotension, individuals with SCI above T6 frequently experience possible life-threatening episodic bouts of hypertension, a disorder known as autonomic dysreflexia (AD) [35]. This condition is not just characterized by episodes of dangerously high BP (more than $20 \mathrm{mmHg}$ systolic BP increase from baseline), but is also commonly accompanied by symptoms like severe headache, sweating, goosebumps, and flushing above the injury level. If unmanaged, these $\mathrm{AD}$ bouts can in rare cases result in seizures, intracranial hemorrhage, stroke, or even death [38-40].

Both noxious and non-noxious stimuli activating sensory or visceral afferents below the lesion could trigger $\mathrm{AD}$. Sensory afferents become activated during cold application, such as during a CPT, or penile vibration [41]. On the other hand, visceral afferents are activated by bladder and bowel distension which are common triggers for AD. While in clinical settings urodynamic procedure is commonly used to trigger $\mathrm{AD}$ under controlled circumstances [42], colorectal distension is the experimental equivalent in rodents [43]. All the different afferent stimuli trigger exaggerated activation of spinal sympathetic circuits caudal to the injury site. This usually results in sympathetically mediated peripheral vasoconstriction in the gut, muscle, and skin vascular beds below the level of lesion, causing extreme systemic hypertension [35]. Finally, a parasympathetic-induced bradycardia could be present during episodes of $\mathrm{AD}$ as a baroreflexmediated response to the preceding hypertension [44]. Although there is still no consensus on the exact pathophysiology explaining $\mathrm{AD}$, it is generally accepted that loss of inhibitory supraspinal input to SPNs probably plays a role in the development of $\mathrm{AD}$ [45]. Other proposed mechanisms include increased nerve growth factor inducing profuse sprouting of central processes of sensory and visceral afferents contributing to exaggerated reflexes [46-49], aberrant plastic changes in propriospinal neurons [46, 50], and peripheral vasculature structural and functional changes including receptor hyper-responsiveness [51, 52].

The following section will address in how far such alterations in cardiovascular physiology can be investigated with the use of a CPT in individuals with different level and severity of injury.

\section{Hemodynamic responses during foot CPT in tetraplegia}

During foot CPT a significant increase in MAP of 14-20 $\mathrm{mmHg}$ has been found in individuals with tetraplegia [6, 7 , $9,10,13]$. In individuals with sensorimotor complete (AIS A) tetraplegia the afferent stimuli from skin thermoreceptors do not reach the thalamus and consequently vasomotor center in the medulla cannot elicit a cold pressure response. The increased BP during foot CPT in individuals with tetraplegia suggests that reflex sympathetic discharge to the peripheral vessels is likely activated through the isolated spinal cord, which is no longer influenced by supraspinal inhibitory pathways (see Fig. 3A, B) [6, 7, 9, 10, 13]. This is probably due to similar mechanism involved during $\mathrm{AD}$ and this hypothesis is reinforced by the fact that plasma norepinephrine, the neurotransmitter released at sympathetic postganglionic endings, is significantly increased during foot CPT [7]. 


A) High-level tetraplegia (C4)
Hand CPT: BP increase, baroreflex-mediated
bradykardia - cold stimulus enters below the
level of lesion and leads to spinal reflexive
discharge of SPNs, comparable to AD.
Foot CPT: BP increase, baroreflex-mediated
bradykardia - spinal reflexive discharge of
SPNs, comparable to AD.
B) Low-level tetraplegia (C7)
Hand CPT: no or only slight BP increase - cold
stimulus enters at the level of lesion and
depending on the integrity of afferents, SPNs
activation is diminished.
Foot CPT: BP increase, baroreflex-mediated
bradykardia - spinal reflexive discharge of
SPNs, comparable to AD.

\section{C) High-level paraplegia (T5)}

Hand CPT: increase in HR, moderate increase in BP - sympathetic activation of heart fully intact, but only upper thoracic part of SPNs contribute to BP increase.

Foot CPT: BP increase, baroreflex-mediated bradykardia, but less pronounced compared to tetraplegic subjects. This is due to preservation of supraspinal control of upper part (T1-T5) of SPNS.

\section{D) Low-level paraplegia (T10)}

Hand CPT: BP and HR increase - physiological CPT response, majority of SPNs activated.

Foot CPT: no BP or HR response - afferent signal is blocked and can not activate enough SPNs to trigger BP increase.

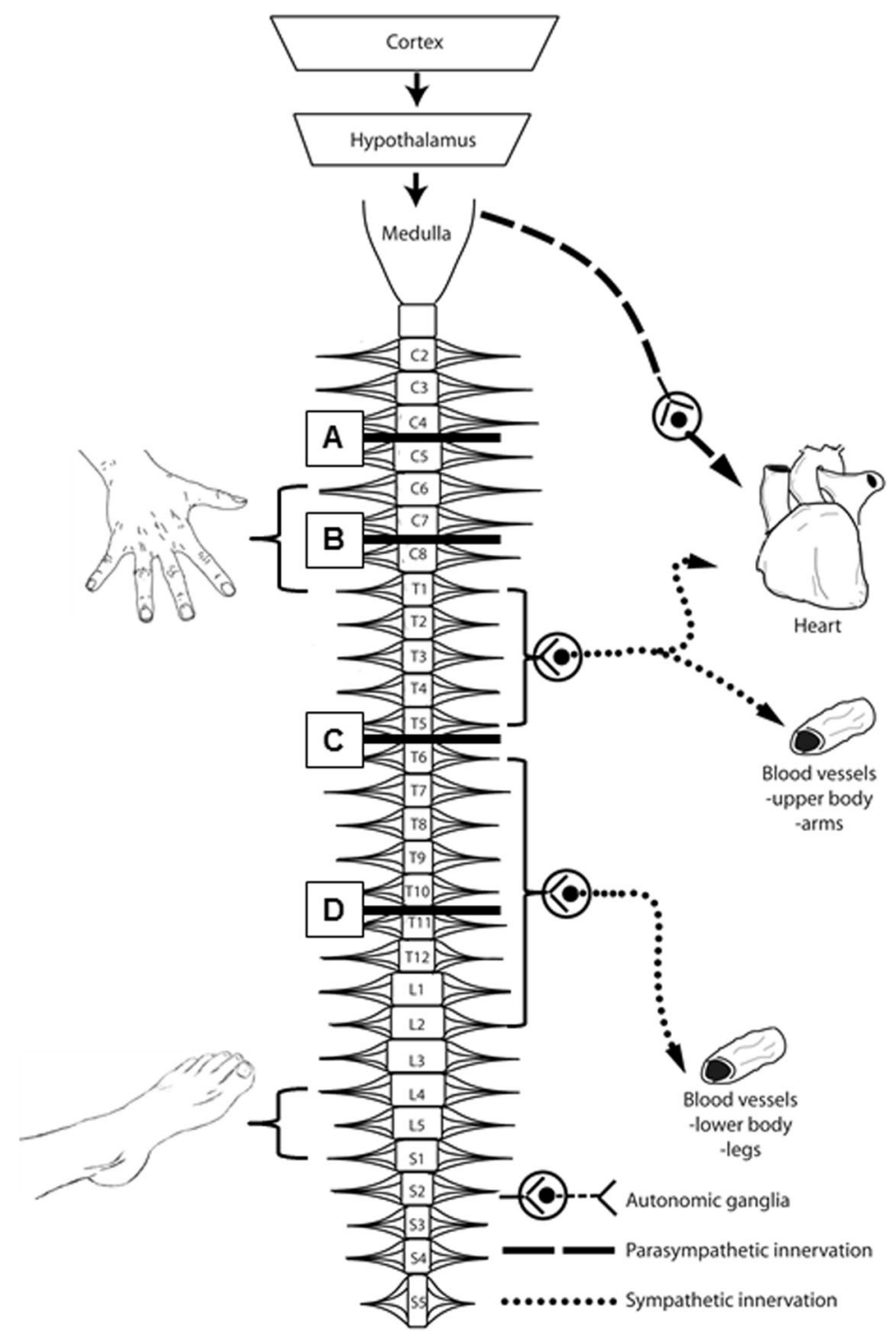

vasculature beds in the gut and lower extremities are under the control of the more caudal T6-L2 spinal SPNs. This segmental sympathetic innervation of blood vessels is particularly important for understanding cardiovascular control in individuals with SCI. Four different cases of CPT responses with underlying mechanisms are described. Hand immersion into cold water (up to the wrist) activates C6-T1 sensory afferents, while foot immersion activates L4-S1 sensory afferents. For all cases sensorimotor as well as autonomic complete SCIs are assumed (with permission from Krassioukov [59])
In terms of $\mathrm{HR}$ responses during foot CPT, studies report either no change in HR [7] or a decrease in HR [6, 9] in individuals with tetraplegia. The decrease in HR was suggested to be due to stimulation of sinoaortic baroreceptors and increased vagal efferent activity, i.e., baroreflex-mediated bradycardia often seen during $\mathrm{AD}$ bouts $[6,9]$. This is in contrast to the rise in HR observed in able-bodied individuals and can be explained by the fact that individuals with high-level SCI have lost or diminished sympathetic supraspinal control over their heart, but intact paraysmpathetic cardiac control through the vagal nerve. On the other hand, a stable HR during foot CPT in tetraplegia was attributed to the balance between reflexive excitation of spinal cardiac sympathetic neurons induced by activation of skin thermoreceptors and parasympathetic input to the heart induced by activation of arterial baroreceptors, which were stimulated by the hypertension during foot cooling [7]. 


\section{Hemodynamic responses during foot CPT in paraplegia}

Hemodynamic responses during foot CPT tend to be less pronounced in individuals with high-level paraplegia compared to those with tetraplegia. Individuals with high-level paraplegia showed an increase in BP of $4 \mathrm{mmHg}$ in SBP [9, 10] and $6 \mathrm{mmHg}$ in MAP [6] during foot CPT. The less pronounced $\mathrm{BP}$ increase was mainly attributed to the fact that although the foot CPT could trigger AD responses, there is a smaller pool of blood vessels with lost supraspinal sympathetic control below the level of injury in individuals with paraplegia (see Fig. 3C). However, supraspinal sympathetic control of the heart and larger pool of blood vessels, that are above the level of injury, is fully preserved [6]. In this regard it is worth to mention that cerebral blood flow velocity showed a prominent increase in the absence of a pronounced BP increase [9]. This suggests that cold information from the foot can still reach blood vessels in the brain through the sympathetic chain and possibly the brainstem vasomotor center and another mechanisms than AD triggers the increased cerebral blood flow [9].

In accordance to the findings in individuals with tetraplegia, a possible baroreflex-mediated bradycardia ( -7 and $-8 \mathrm{bpm}$ ) during foot CPT has also been found in individuals with high-level paraplegia [6]. However, since a pronounced increase in BP is missing, Catz et al. [9] suggested another mechanism other than baroreflex-mediated bradycardia or physiological CPT response.

One study conducted in individuals with Brown-Séquard syndrome highlighted the importance of intact afferent pathways as a crucial component for BP and HR changes during foot CPT. When the foot with intact sensation was exposed to cold, MAP and HR increased $(23 \mathrm{mmHg}$, $25 \mathrm{bpm}$ ), while when the foot with a sensory deficit was exposed to the cold stimulus, no increase in BP or HR was observed [11]. The increase in MAP and HR is attributed to a physiological cold pressor response, while the increase in $\mathrm{BP}$ observed during foot $\mathrm{CPT}$ in individuals with tetraplegia is attributed to $\mathrm{AD}$, as described above.

\section{Hemodynamic responses during hand CPT in tetraplegia}

The hemodynamic outcome during hand CPT in individuals with tetraplegia is more variable compared during foot CPT. Most studies did not report any significant changes in BP or HR during hand CPT in individuals with tetraplegia [10, 12, 13]. They concluded that in these cases the hand CPT represents a stimulus above lesion and the cold stimulus could not evoke any activation of thoracic sympathetic outflow as a major part of SPNs are located below the level of injury.
On the other hand, Catz et al. (2007) showed an MAP increase of $9 \mathrm{mmHg}$ and an $\mathrm{HR}$ increase of $4 \mathrm{bpm}$ during hand CPT. The investigators suggested that the underlying mechanism responsible for the $\mathrm{BP}$ increase in individuals with tetraplegia is the same as during foot $\mathrm{CPT}$ and has been attributed to elicited AD [8]. Additionally, Groothuis and Hopman [53] suggested that in individuals with lower tetraplegia with spinal cord lesion at $\mathrm{C} 6-\mathrm{C} 8$ might interfere with afferent fibers integrity, e.g., direct injury to the dorsal roots afferents, such as that afferent noxious cold information is not transmitted to the spinal cord, and therefore the $\mathrm{BP}$ increase during hand CPT was not as high as usually seen during $\mathrm{AD}$ (see Fig. 3B).

\section{Hemodynamic responses during hand CPT in paraplegia}

In individuals with high-level paraplegia, usually moderate increases in BP (7 mmHg SBP [10], $5 \mathrm{mmHg}$ MAP [8]) have been reported during hand CPT. A hand CPT in highlevel paraplegia (T4-6) elevates sympathetic activity above lesion level and, therefore, led to increased HR (9 bpm) and cardiac contractility [8], but the splanchnic and lower limb vascular beds are innervated below the spinal cord lesion, i.e., from T6-L2 spinal levels (see Fig. 3C) and therefore BP only marginally increased in these individuals [53].

In contrast, an increase of BP and HR has been reported in individuals with low-level paraplegia (T10-L1) [12], which reflects a normal physiological CPT response (see Fig. 3D).

\section{CPT and the integrity of autonomic pathways}

Presently, the International Standards for Neurological Classification of SCI are employed to assess the completness and severity of injury to sensory and motor pathways following an SCI [54]. Yet, these standards do not necessarily correspond with the completeness and severity of the injury of spinal autonomic pathways [55]. Although, the international SCI community made significant advances towards the autonomic evaluation within the last decade by developing international standards to document remaining autonomic functions after SCI [56], the field is still lacking established validated methods for measuring the severity and completeness of spinal autonomic pathways following SCI [10]. For instances, sympathetic skin responses (SSRs) were suggested to be used in conjunction with other validated autonomic tests in order to predict or document autonomic dysfunction in SCI [57]. SSRs measure only sudomotor cholinergic function and, although closely linked anatomically with vasomotor pathways [58], may not be 
Table 2 Different CPT protocols

\begin{tabular}{llll}
\hline Author & CPT & Water temperature & Duration of immersion (seconds) \\
\hline Ogata et al. (2012) & Foot & $2.7 / 2.9^{\circ} \mathrm{C}$ & 180 \\
Previnaire et al. (2012) & Hand/foot & Crushed ice & $90-120$ \\
Catz et al. (2008) & Hand & Ice water & $40-150$ \\
Catz et al. (2007) & Foot & Ice water & $40-120$ \\
Mizushima et al. (2003) & Foot & $0{ }^{\circ} \mathrm{C}$ & 180 \\
Winchester et al. (2000) & Foot & $0-2{ }^{\circ} \mathrm{C}$ & 120 \\
Lehmann et al. (1989) & Hand/forehead & Ice water & 60 \\
Pollock et al. (1950) & Hand/foot & $4{ }^{\circ} \mathrm{C}$ & $30-60$ \\
\hline
\end{tabular}

representative of impairment of the entirety of sympathetic cardiovascular outflow. Additional testing of adrenergic, vasomotor function may be required to assess the completeness of autonomic pathways following an SCI. The CPT might play an important role in this regard.

Several studies assessed the integrity of the autonomic nervous system with the help of a hand CPT [10, 12, 14]. Lehmann et al. (1989), for instances, showed BP and HR increases in a low-level injury group (T10-L1) as expected. However, neither of these hemodynamic parameters varied statistically from baseline in a high-level injury group (C5-C8) [12]. The authors therefore suggested a total disruption of sympathetic outflow due to an autonomic complete SCI in the high-level injury group.

In contrast, individuals with comparable cervical lesion levels (C4-C7) have shown a significant increase in BP during hand CPT [8]. This finding has been discussed to stem from elicitation of AD or from a possible autonomic incomplete lesion [8].

Previnaire et al. (2012) only found a slight, nonsignificant increase in $\mathrm{BP}(5 \mathrm{mmHg} \mathrm{SBP})$ during hand $\mathrm{CPT}$ in individuals with tetraplegia. This increase in BP in individuals with sensorimotor complete (AIS A) tetraplegia was unexpected for the authors and therefore they concluded that hand CPT is of limited interest in the assessments of a complete sympathetic lesion. On the other hand, the same group proposed the foot CPT as a valuable tool to assess the integrity of autonomic pathways, as in individuals with high-level paraplegia and tetraplegia reflex activity of the isolated spinal cord, i.e., $\mathrm{AD}$, has been shown [10].

It is important to mention that a significant increase in $\mathrm{BP}$ during foot CPT might not necessarily reflect $\mathrm{AD}$, but maybe to the existence of an incomplete lesion of autonomic vasomotor pathways. In the latter case, the increase of BP just reflects a physiological cold pressor response as observed in able-bodied individuals. In such cases HR responses might provide additional information about the autonomic completeness, such as bradycardia would reflect an autonomic complete while tachycardia an autonomic incomplete injury.

\section{Methodological considerations}

A closer look at the different CPT protocols used in the SCI population highlights the methodological discrepancies. Not only water temperature $\left(0-4{ }^{\circ} \mathrm{C}\right)$ was different among the studies, but especially the exposure time (40-180 s) of the individuals to the cold stimulus differed (see Table 2). BP was either continuously or discretely monitored, with intervals in discrete monitoring from $15-60 \mathrm{~s}$. All these variations might bias the results and thus cautious comparisons between studies are required. In addition, there is a need for good standardized CPT protocols in individuals with SCI.

\section{Conclusion}

The practicability of the CPT to assess sympathetic vasomotor function in individuals with SCI is not clear-cut. The interpretation of CPTs seems to be challenging in this patient population as it shows highly variable outcomes depending on the level of spinal lesion as well as location of cold application (as summarized in Fig. 3). In order to at least standardize the test protocols, we would suggest immersing the hand or foot for at least 2 min into cold water $\left(\leq 4{ }^{\circ} \mathrm{C}\right)$ with continuous BP and HR monitoring.

In general, further investigations in a wider range of individuals with SCI, i.e., incomplete injuries, might be helpful to examine the ability of CPT assessing the integrity of autonomic pathways. A combination of stimuli above and below the lesion as well as a selection of additional test of different autonomic systems might be needed when consider investigating such a multifaceted system as the autonomic nervous system.

Acknowledgements The authors are grateful to the Swiss National Science Foundation for providing an advanced postdoc mobility fellowship to MH. Research in AVK's laboratory is supported by grants from the Canadian Institutes of Health Research and the Craig. H Neilsen Foundation. 


\section{Compliance with ethical standards}

Conflicts of interest The authors declare that they have no conflict of interest.

\section{References}

1. Claydon VE, Krassioukov AV. Orthostatic hypotension and autonomic pathways after spinal cord injury. J Neurotrauma. 2006;23:1713-25.

2. Handrakis JP, DeMeersman RE, Rosado-Rivera D, LaFountaine MF, Spungen AM, Bauman WA, et al. Effect of hypotensive challenge on systemic hemodynamics and cerebral blood flow in persons with tetraplegia. Clin Auton Res. 2009;19:39-45.

3. Miyatani M, Masani K, Oh PI, Miyachi M, Popovic MR, Craven BC. Pulse wave velocity for assessment of arterial stiffness among people with spinal cord injury: a pilot study. J Spine Cord Med. 2009;32:72-8.

4. Freeman R. Assessment of cardiovascular autonomic function. Clin Neurophysiol. 2006;117:716-30.

5. Scharf M, Korczyn AD. Cold pressor test. In: Korczyn AD, editor. Handbook of Autonomic Nervous System Dysfunction. New York: Marcel Decker; 1995. p. 557-62.

6. Ogata $\mathrm{H}$, Hobara $\mathrm{H}$, Uematsu A, Ogata T. Limb oxygenation during the cold pressor test in spinal cord-injured humans. Clin Auton Res. 2012;22:71-8.

7. Mizushima T, Tajima F, Okawa H, Umezu Y, Furusawa K, Ogata H. Cardiovascular and endocrine responses during the cold pressor test in subjects with cervical spinal cord injuries. Arch Phys Med Rehabil. 2003;84:112-8.

8. Catz A, Bluvshtein V, Pinhas I, Akselrod S, Gelernter I, Nissel T, et al. Cold pressor test in tetraplegia and paraplegia suggests an independent role of the thoracic spinal cord in the hemodynamic responses to cold. Spinal Cord. 2008;46:33-8.

9. Catz A, Bluvshtein V, Korczyn AD, Pinhas I, Gelernter I, Nissel $\mathrm{T}$, et al. Modified cold pressor test by cold application to the foot after spinal cord injury: suggestion of hemodynamic control by the spinal cord. Am J Phys Med Rehabil. 2007;86:875-82.

10. Previnaire JG, Soler JM, Leclercq V, Denys P. Severity of autonomic dysfunction in patients with complete spinal cord injury. Clin Auton Res. 2012;22:9-15.

11. Winchester PK, Williamson JW, Mitchell JH. Cardiovascular responses to static exercise in patients with Brown-Sequard syndrome. J Physiol. 2000;527(Pt 1):193-202.

12. Lehmann KG, Shandling AH, Yusi AU, Froelicher VF. Altered ventricular repolarization in central sympathetic dysfunction associated with spinal cord injury. Am J Cardiol. 1989;63: 1498-504.

13. Pollock LJ, Boshes B, Chor H, Finkelman I, Arieff AJ, Brown M. Defects in regulatory mechanisms of autonomic function in injuries to spinal cord. J Neurophysiol. 1951;14:85-93.

14. Kooijman M, Rongen GA, Smits P, Hopman MT. Preserved alpha-adrenergic tone in the leg vascular bed of spinal cordinjured individuals. Circulation. 2003;108:2361-7.

15. Greene MA, Boltax AJ, Lustig GA, Rogow E. Circulatory dynamics during the cold pressor test. Am J Cardiol. 1965;16: $54-60$.

16. Lovallo W. The cold pressor test and autonomic function: a review and integration. Psychophysiology. 1975;12:268-82.

17. Nakamura T, Kawabe K, Sapru HN. Cold pressor test in the rat: medullary and spinal pathways and neurotransmitters. Am J Physiol Heart Circ Physiol. 2008;295:H1780-7.

18. Lebedev VP, Krasyukov AV, Nikitin SA. Electrophysiological study of sympathoexcitatory structures of the bulbar ventrolateral surface as related to vasomotor regulation. Neuroscience. 1986;17:189-203.

19. Krassioukov A, Weaver LC. Anatomy of the autonomic nervous system. In: Teasell RW, Baskerville VB, editors. Physical Medicine and Rehabilitation: State of the Art Reviews. Philadelphia: Hanley \& Belfus, Inc.; 1996. p. 1-14.

20. Phillips AA, Krassioukov AV, Ainslie PN, Warburton DE. Baroreflex function after spinal cord injury. $\mathrm{J}$ Neurotrauma. 2012;29:2431-45.

21. Cui J, Wilson TE, Crandall CG. Baroreflex modulation of muscle sympathetic nerve activity during cold pressor test in humans. Am J Physiol Heart Circ Physiol. 2002;282:H1717-23.

22. Pang CC. Autonomic control of the venous system in health and disease: effects of drugs. Pharmacol Ther. 2001;90:179-230.

23. Oblak JP, Zaletel M, Zvan B, Kiauta T, Pogacnik T. The effect of age on cerebrovascular reactivity to cold pressor test and head-up tilt. Acta Neurol Scand. 2002;106:30-3.

24. Korhonen I. Blood pressure and heart rate responses in men exposed to arm and leg cold pressor tests and whole-body cold exposure. Int J Circumpolar Health. 2006;65:178-84.

25. Saab PG, Llabre MM, Hurwitz BE, Schneiderman N, Wohlgemuth W, Durel LA, et al. The cold pressor test: vascular and myocardial response patterns and their stability. Psychophysiology. 1993;30:366-73.

26. Tsai SJ, Chen SC, Leu TM, Chen CM, Chou HH, Peng HY, et al. Impairment of cerebral hemodynamic response to the cold pressor test in patients with Parkinson's disease. Park Relat Disord. 2009;15:94-100

27. Wakabayashi K, Takahashi H. Neuropathology of autonomic nervous system in Parkinson's disease. Eur Neurol. 1997;38:2-7.

28. Ziemssen T, Reichmann H. Cardiovascular autonomic dysfunction in Parkinson's disease. J Neurol Sci. 2010;289:74-80.

29. Adamec I, Habek M. Autonomic dysfunction in multiple sclerosis. Clin Neurol Neurosurg. 2013;115:73-8.

30. Vita G, Fazio MC, Milone S, Blandino A, Salvi L, Messina C. Cardiovascular autonomic dysfunction in multiple sclerosis is likely related to brainstem lesions. J Neurol Sci. 1993;120:82-6.

31. Thomaides TN, Zoukos Y, Chaudhuri KR, Mathias CJ. Physiological assessment of aspects of autonomic function in patients with secondary progressive multiple sclerosis. J Neurol. 1993;240:139-43.

32. Burgos LG, Ebert TJ, Asiddao C, Turner LA, Pattison CZ, WangCheng $\mathrm{R}$, et al. Increased intraoperative cardiovascular morbidity in diabetics with autonomic neuropathy. Anesthesiology. 1989;70:591-7.

33. Shapiro AP, Moutsos SE, Krifcher E. Patterns of pressor response to noxious stimuli in normal, hypertensive, and diabetic subjects. J Clin Invest. 1963;42:1890-8.

34. Orlov S, Bril V, Orszag A, Perkins BA. Heart rate variability and sensorimotor polyneuropathy in type 1 diabetes. Diabetes Care. 2012;35:809-16.

35. Krassioukov A, Claydon VE. The clinical problems in cardiovascular control following spinal cord injury: an overview. Prog Brain Res. 2006;152:223-9.

36. West CR, Alyahya A, Laher I, Krassioukov A. Peripheral vascular function in spinal cord injury: a systematic review. Spinal Cord. 2013;51:10-19.

37. West CR, Mills P, Krassioukov AV. Influence of the neurological level of spinal cord injury on cardiovascular outcomes in humans: a meta-analysis. Spinal Cord. 2012;50:484-92.

38. Ho CP, Krassioukov AV. Autonomic dysreflexia and myocardial ischemia. Spinal Cord. 2010;48:714-5.

39. Eltorai I, Kim R, Vulpe M, Kasravi H, Ho W. Fatal cerebral hemorrhage due to autonomic dysreflexia in a tetraplegic patient: case report and review. Paraplegia. 1992;30:355-60. 
40. Wan D, Krassioukov AV. Life-threatening outcomes associated with autonomic dysreflexia: a clinical review. J Spinal Cord Med. 2014;37:2-10.

41. Sheel AW, Krassioukov AV, Inglis JT, Elliott SL. Autonomic dysreflexia during sperm retrieval in spinal cord injury: influence of lesion level and sildenafil citrate. J Appl Physiol (1985). 2005;99:53-8.

42. Giannantoni A, Di Stasi SM, Scivoletto G, Mollo A, Silecchia A, Fuoco U, et al Autonomic dysreflexia during urodynamics. Spinal Cord. 1998;36:756-60.

43. Mayorov DN, Adams MA, Krassioukov AV. Telemetric blood pressure monitoring in conscious rats before and after compression injury of spinal cord. J Neurotrauma. 2001;18:727-36.

44. Teasell RW, Arnold JM, Krassioukov A, Delaney GA. Cardiovascular consequences of loss of supraspinal control of the sympathetic nervous system after spinal cord injury. Arch Phys Med Rehabil. 2000;81:506-16.

45. Krassioukov AV, Bunge RP, Pucket WR, Bygrave MA. The changes in human spinal sympathetic preganglionic neurons after spinal cord injury. Spinal Cord. 1999;37:6-13.

46. Hou S, Duale H, Cameron AA, Abshire SM, Lyttle TS, Rabchevsky AG. Plasticity of lumbosacral propriospinal neurons is associated with the development of autonomic dysreflexia after thoracic spinal cord transection. J Comp Neurol. 2008;509: 382-99.

47. Weaver LC, Verghese P, Bruce JC, Fehlings MG, Krenz NR, Marsh DR. Autonomic dysreflexia and primary afferent sprouting after clip-compression injury of the rat spinal cord. J Neurotrauma. 2001;18:1107-19.

48. Brown A, Ricci MJ, Weaver LC. NGF message and protein distribution in the injured rat spinal cord. Exp Neurol. 2004;188: $115-27$.

49. Krenz NR, Meakin SO, Krassioukov AV, Weaver LC. Neutralizing intraspinal nerve growth factor blocks autonomic dysreflexia caused by spinal cord injury. J Neurosci. 1999;19:7405-14.
50. Krassioukov AV, Johns DG, Schramm LP. Sensitivity of sympathetically correlated spinal interneurons, renal sympathetic nerve activity, and arterial pressure to somatic and visceral stimuli after chronic spinal injury. J Neurotrauma. 2002;19:1521-9.

51. Brock JA, Yeoh M, McLachlan EM. Enhanced neurally evoked responses and inhibition of norepinephrine reuptake in rat mesenteric arteries after spinal transection. Am J Physiol Heart Circ Physiol. 2006;290:H398-405.

52. Phillips AA, Matin N, Frias B, Zheng MM, Jia M, West C, et al Rigid and remodelled: cerebrovascular structure and function after experimental high-thoracic spinal cord transection. J Physiol. 2016;594:1677-88.

53. Groothuis JT, Hopman MT. Hemodynamic responses to the cold pressor test in spinal cord-injured individuals; control of the splanchnic vascular bed is the key factor. Spinal Cord. 2009;47:95. Author reply 96.

54. Kirshblum SC, Burns SP, Biering-Sorensen F, Donovan W, Graves DE, Jha A, et al. International standards for neurological classification of spinal cord injury (revised 2011). J Spinal Cord Med. 2011;34:535-46.

55. West CR, Bellantoni A, Krassioukov AV. Cardiovascular function in individuals with incomplete spinal cord injury: a systematic review. Top Spinal Cord Inj Rehabil. 2013;19:267-78.

56. Krassioukov A, Biering-Sorensen F, Donovan W, Kennelly M, Kirshblum S, Krogh K, et al. International standards to document remaining autonomic function after spinal cord injury. J Spinal Cord Med. 2012;35:201-10.

57. Berger MJ, Hubli M, Krassioukov AV. Sympathetic skin responses and autonomic dysfunction in spinal cord injury. $\mathrm{J}$ Neurotrauma. 2014;31:1531-9.

58. Nathan PW, Smith MC. The location of descending fibres to sympathetic preganglionic vasomotor and sudomotor neurons in man. J Neurol Neurosurg Psychiatry. 1987;50:1253-62.

59. Krassioukov A. Autonomic function following cervical spinal cord injury. Respir Physiol Neurobiol. 2009;169:157-64. 